\title{
Improved Outcomes in Paediatric Intestinal Failure with Aggressive Prevention of Liver Disease
}

Authors

Affiliations

\author{
D. Sigalet ${ }^{1}$, D. Boctor ${ }^{2}$, M. Robertson ${ }^{2}$, V. Lam ${ }^{3}$, M. Brindle ${ }^{4}$, K. Sarkhosh ${ }^{4}$, L. Driedger ${ }^{2}$, M. Sajedi ${ }^{2}$
}

\author{
${ }^{1}$ Alberta Children's Hospital/University of Calgary, Pediatric General Surgery, Calgary, Canada \\ ${ }^{2}$ Alberta Children's Hospital, Department of Pediatrics, Calgary, Canada \\ ${ }^{3}$ Alberta Children's Hospital, Pediatric Surgery, Calgary, Canada \\ ${ }^{4}$ Alberta Children's Hospital, Pediatric General Surgery, Calgary, Canada
}

\author{
Key words \\ - TPN \\ - Omegevan \\ - short bowel syndrome \\ - STEP procedure \\ - bacterial \\ - overgrowth
}

received May 24, 2009 accepted after revision August 22, 2009

\section{Bibliography}

DOI $10.1055 / \mathrm{s}-0029-1241865$ Published ahead of print: 28 October 2009

Eur J Pediatr Surg 2009; 19: 348-353 ๔ Georg Thieme Verlag KG Stuttgart · New York ISSN 0939-7248

Correspondence

\section{Prof. David Sigalet}

Alberta Children's Hospital/

University of Calgary

Pediatric General Surgery

2888 Shaganappi Trail NW

T3B 6A8 Calgary

Canada

sigalet@ucalgary.ca

\section{Abstract \\ V}

Background/Purpose: A protocol-driven care algorithm for the care of intestinal failure (IF) centred on therapies to prevent Parenteral Nutrition Associated Cholestasis (PNAC) was instituted in 2006. We report our results from 2006-2009, and compare them to the outcomes of our previous cohort of patients (1998-2006).

Methods: With regional ethics board approval, we have been prospectively gathering data on patient with IF cared for by our regional surgical unit. IF was defined as a residual bowel length of $<40 \mathrm{~cm}$ or a requirement for PN for greater than 60 days. With the development of a multidisciplinary care team, a protocol-driven strategy to prevent PNAC was instituted in 2006, with aggressive introduction of enteral feeds, use of prophylactic antibiotics to prevent bacterial overgrowth, lipid reduction and use of a fish oil-derived lipid preparation for cholestasis and Serial Transverse Enteroplasty (STEP) if bowel dilation occurred.

Results: In the era from 1998-2006, 33 patients were identified, with a $72 \%$ survival; the direct bilirubin averaged $112 \pm 34 \mu \mathrm{M} / \mathrm{L}$ after 3 months of PN. 8/33 (27\%) of patients received

\section{Introduction}

Intestinal failure is a challenging clinical problem in the paediatric population. The ability to provide appropriate calories via parenteral nutrition provides a safety net for the clinician $[1,2]$. However in the infant population this is a doubleedged sword. Historically, the use of parenteral nutrition for periods greater than 6-8 weeks would predictably result in the onset of parenteral nutrition-associated liver disease (PNALD) $[3,4]$. In the past, the complication of progressive hepatic fibrosis and liver failure was a constant prophylactic antibiotics, and none received fish oil-based lipids. The most common causes of IF were gastroschisis (30\%) and atresia (21\%); 31 of 33 patients were infants. Average time to intestinal rehabilitation/death was $4.5 \pm 3$ months. All deaths were related to sepsis or PN/liver failure. In the era from 2006-2009, 22 patients have been followed, with $100 \%$ survival $^{*}$. Average bilirubin after 3 months of PN was $8 \pm 2.2 \mu \mathrm{M} / \mathrm{L}^{*}, 20 / 22$ $(90 \%)^{*}$ received prophylactic antibiotics, and 6/22(27\%)* received fish oil-based lipid PN. The common causes of IF were gastroschisis 15/22 (68\%) and atresia (27\%). 18/22 are weaned from $\mathrm{PN}$, and the average time to intestinal rehabilitation was $2.7 \pm 1.3$ months, 4 patients underwent STEP procedures. $\left({ }^{*} \mathrm{p}<0.05\right.$ by Fischer's exact or Student's t-test, data mean \pm SD).

Conclusions: The institution of an aggressive protocol of advancing enteric feeds, oral antibiotic prophylaxis for bacterial overgrowth, fish oil-based lipid use, and the STEP procedure for dilated bowel has resulted in an apparent increase in survival and a remarkable improvement in liver function in a paediatric IF population. Further studies to define the relative importance of these therapies are recommended.

threat for these patients. There is an inverse relationship between the length of bowel and the frequency of liver failure [1,5-8]. This is further exacerbated by episodes of line or bacterial overgrowth-induced sepsis [9-11].

In this context the ability to provide nutrition by the enteral route is the ultimate treatment for PNALD $[12,13]$. However, in the drive to provide calories by the enteral route the potential for promoting bacterial overgrowth within the small bowel is a continual threat. Episodes of sepsis definitely increase the rate and the severity of liver disease. Thus the clinical care of these 
patients consists of a series of small steps to increase enteral nutrition while continually watching for problems with bacterial overgrowth, or recurrent necrotising enterocolitis.

Certain key observations by clinical groups over the recent years have helped refine the general strategy for the care of these patients. Georgeson observed that the aggressive use of rotating antibiotics appeared to reduce the risk of gut-driven sepsis, and also had a beneficial or protective effect on the progress of liver disease [14]. While other groups have had variable results, this concept is theoretically quite logical. It has been shown that the short gut has increased permeability; factors which increase the growth of bacteria within the intestinal lumen increase the exposure of the liver either to intact bacteria or to their breakdown components, which then may worsen cholestasis $[1,9,15]$.

In addition, it has been suggested that the provision of calories in the form of fat may also contribute to the development of liver disease. This has led to suggestions of using a lipid sparing or lipid reduction strategy, if cholestasis is apparent $[1,2,16]$. In a continuation of this theme, the description of the beneficial effects of fish oil-based lipid preparations with an increase of $\omega-3$ omega fatty acids has also been shown to have an apparent benefit in reversing established PN-associated cholestasis [1719].

Finally, in terms of positive therapies to improve the tolerance of enteral feeds, the use of a serial transverse enteroplasty (STEP) procedure to increase the useful surface area, while coincidentally reducing the tendency to bacterial overgrowth, has shown benefits in selected patients $[20,21]$.

Our surgical unit has had a long standing interest in the treatment of Short Bowel Syndrome. In the years between 1998 and 2006, despite this interest, there was only limited coordination of the care of infants following intestinal resection or gastroschisis repair. Nutritional support was provided, with attention focused on the provision of adequate calories, primarily by PN, and the institution of enteral feeds was primarily at the discretion of the attending surgeon. Coincident with a move of the hospital in 2006, we were able to institute a cohesive multidisciplinary team approach (CHIRP) to care for patients with intestinal failure [6,22]. This occurred in October 2006. All infants undergoing intestinal resection or repair of gastroschisis came under the care of the CHIRP team, as well as older children on long-term PN (longer than 60 days).

At that time, a more aggressive policy of increasing enteral feeds and the use of rotating antibiotics with a promotility agent was instituted (www.ucalgary/pediatricsurgery/CHIRP protocol). In brief, nutrition was initially provided as TPN, following conventional guidelines for total calories and the allocation of fat, protein and carbohydrates $[6,23]$.This included the provision of calories appropriate for age and weight, following a "balanced" PN approach with fat prescribed at a rate of $2-3 \mathrm{~g} / \mathrm{kg}$ per day given as a conventional $20 \%$ Intralipid emulsion (Fresenius Kabi Canada, Toronto, ON, Canada). Once signs of bowel function returned post-operation (passage of stool, soft abdomen, bowel sounds present), feeds of expressed breast milk (EBM) were commenced at $10 \mathrm{cc} / \mathrm{kg} /$ day via continuous nasogastric tube feeds. If EBM was not available, then Neocate (Nutricia NA, St Laurent, QC, Canada) was used to minimise the potential for allergic enteropathy. Feeds were increased by $10 \mathrm{cc} / \mathrm{kg} / \mathrm{day}$, unless signs of distension or increased gastric residuals occurred. Absorption was monitored with a twice weekly determination of reducing substances and faecal fat determination. As feeds increased, PN was decreased, aiming to keep growth in the 5$10^{\text {th }}$ percentile. Once PN rates decreased below $15 \mathrm{ml} /$ hour, PN was cycled.

If there was any delay in the tolerance of enteral feeds, i.e. distension or increased gastric residuals, for more than 3-4 days, then a promotility agent was used (metoclopromide, $10 \mathrm{mg} / \mathrm{kg}$ IV divided qid). If feeding intolerance persisted, then rotating antibiotics were begun: gentamycin $5 \mathrm{mg} / \mathrm{kg} /$ day, given b.i.d. via the nasogastric tube, for one week, followed by one week off antibiotics, then metronidazole $20 \mathrm{mg} / \mathrm{kg} /$ day, given in 2 doses via the nasogastric tube for one week, followed by another week off antibiotics, after which the cycle commenced again. In cases of significant bowel distension or suspected bacterial overgrowth clinically, then the one week off antibiotics was omitted, and continuously alternating cycles of gentamycin and flagyl were used.

If biochemical signs of liver failure ensued (conjugated blilirubin over $50 \mu \mathrm{mol} / \mathrm{L}$ in infants older than 2 months of age), then an aggressive lipid reduction strategy was started, with a reduction in intravenous lipids (Intralipid, $1.0 \mathrm{~g} / \mathrm{kg} /$ day, Frenesius Kabi, Toronto, ON, Canada). If bilirubin climbed above $60 \mu \mathrm{mol} / \mathrm{L}$, then Intralipid was discontinued, and a regimen using a fish oil-based lipid preparation (Omegevan, Frenesius Kabi, by special access, Health Canada) was instituted $(1.0 \mathrm{~g} / \mathrm{kg} /$ day $)$, with monthly monitoring of serum essential fatty acid levels.

Patients underwent routine imaging of the small intestine with an upper GI barium study and follow through, every 6 months for the first 2 years, and annually thereafter, to monitor for the development of distended "overadapted" segments. Imaging was also done if there was a loss of tolerance of enteral feeds or an episode of sepsis with intestinal organisms. If areas of dilated bowel were detected in the setting of a loss of tolerance of enteral feeds, then a STEP procedure was recommended to families. In all cases where this was suggested, the families agreed to surgery.

This report details our experiences in the years 1998-2006 as one distinct phase, in which a "conventional" approach to PN and enteral therapy was used. This is contrasted with the second phase from 2006 to early 2009, when the more aggressive, teambased approach to management was begun, with a focus on increasing enteral feeds, and preventing cholestasis and liver disease. The change in strategy forms a natural break point for comparisons.

\section{Methods \\ $\nabla$}

\section{Study Population}

Infants referred for surgical and nutritional care to the Alberta Children's Hospital between 1 January 1998 and September 2006 form the first cohort in the study, while infants cared for during the time from October 2006 to January 2009 form the second cohort. Data on the original patient demographics, disease type, therapy and progress of feeding was gathered prospectively. Informed consent was obtained from the subject's parents. The protocol for gathering data was approved by the conjoined Regional Health Ethics Board (protocol 16506).

\section{Definition of Terms}

Intestinal failure was defined to have occurred in any child undergoing a laparotomy who 1) was left with less than $40 \mathrm{~cm}$ small bowel length, or 2) required parenteral nutrition after 
laparotomy for more than 60 days. This follows our original definition proposed for surgical cases, but increases the time required for PN support as suggested in recent NIH Paediatric Intestinal Failure Consortium Guidelines (PIF-Con) [4]. Patients with major cardiac or chromosomal anomalies that precluded initial surgical management or prevented an approach with conventional care were excluded. Patients dying in the acute phase of NEC (before considering the start of enteral feeds) were also excluded.

\section{Data Collection}

In the first cohort, from 1998 to 2000, patients were identified by a review of the parenteral nutrition records of the pharmacy, and demographic and feeding data were collected retrospectively. From 2000-2005, patients were identified and followed up prospectively; some elements of data collection were done prospectively, and some elements (lab values and feeding protocols) were collected prospectively. In the recent cohort, data was collected prospectively from the weekly patient chart reviews and from hospital records. Data was entered on a separate spread sheet, and analysed using Prism statistical software (Prism, La Jolla, CA, USA). Outcomes were calculated from the time of the original surgery.

Data collection included initial patient demographics, gestational age, birth weight, and the primary pathology. The surgical procedure was recorded and the length of bowel measured. The percentage of measured bowel length compared with the predicted bowel length based on a published algorithm was calculated [24]. In cases of gastroschisis, an accurate measure of bowel length could not be performed, and so the pre-resection length was assumed to be equivalent to the norms for gestational age and weight.

Data relating to the ongoing clinical status, including tolerance of enteral feeds, were recorded weekly. Biochemical parameters were also collected weekly as part of the routine of parental nutritional monitoring, and included conventional blood counts, electrolytes and liver function tests.

The use of medications including promotility agents, rotating antibiotics, and other medications was recorded. Operative details regarding the STEP procedure, if performed, were also recorded.

\section{Results}

$\nabla$

The majority of patients treated over both time periods were infants, presenting with intestinal failure in the neonatal period ( 0 Table 1). There were no significant differences in the patients' average age, weight or gestational age at surgery. There was a difference in the number of patients presenting with gastroschisis, which increased in the second phase, with a corresponding reduction in the number of babies having necrotising enterocolitis.

The changes in treatments used, according to era, are listed in - Table 2. There was a clearly significant increase in the use of rotating antibiotics, as well as of the strategies invoked to preserve liver function, that is lipid reduction, and the use of a fish oil-based lipid preparation in the recent cohort. Finally, use of the STEP procedure has increased in the recent era.

Outcomes are listed in 0 Table 3. The most striking change is in survival, which improved significantly in the recent cohort (2006-2009: survival was 22 out of 22 vs. 1998-2005: 24 out of
Table 1 Demographics.

\begin{tabular}{|c|c|c|c|}
\hline Parameters & 1998-2006 & 2006-2009 & p-value \\
\hline number of patients & 33 & 22 & \\
\hline birth weight (grams) & $2480 \pm 600$ & $2340 \pm 760$ & NS \\
\hline gestational age (weeks) & $33.7 \pm 3.4$ & $37.1 \pm 5.9$ & NS \\
\hline $\begin{array}{l}\text { gestational age at initial } \\
\text { surgery }\end{array}$ & $35 \pm 12$ & $39 \pm 6.5$ & NS \\
\hline small bowel length $(\mathrm{cm})$ & $59 \pm 33$ & $87 \pm 47$ & NS \\
\hline $\begin{array}{l}\text { small bowel length (\% } \\
\text { predicted (from [35]) }\end{array}$ & $62 \pm 33$ & $72 \pm 31$ & NS \\
\hline ileocaecal valve resected & $7 / 33$ & $6 / 22$ & NS \\
\hline colon resected & $11 / 33$ & $5 / 22$ & NS \\
\hline \multicolumn{4}{|l|}{ diagnosis } \\
\hline gastroschisis & $10(30 \%)$ & $15(68 \%)$ & 0.01 \\
\hline atresia/Atresia + Gsc & $7 / 4(21 \%)$ & $6 / 2(27 \%)$ & NS \\
\hline NEC & $11(30 \%)$ & $3(13 \%)$ & 0.03 \\
\hline other & $5(15 \%)$ & $2(9 \%)$ & NS \\
\hline
\end{tabular}

Data: mean \pm standard deviation. p-values by Fisher's exact test.

Table 2 Treatments by era.

\begin{tabular}{llll} 
Parameter & $\mathbf{1 9 9 8 - 2 0 0 6}$ & $\mathbf{2 0 0 6 - 2 0 0 9}$ & p-value \\
rotating antibiotics & $8 / 33$ & $\mathbf{2 0 / 2 2}$ & 0.01 \\
\hline lipid reduction strategy & $2 / 33$ & $8 / 22$ & 0.04 \\
fish oil-based lipids & $0 / 32$ & $6 / 22$ & 0.001 \\
STEP procedure & $0 / 33$ & $4 / 22$ & 0.03
\end{tabular}

Data: subjects receiving intervention/total subjects in the group. p-values by Fisher's exact test

\begin{tabular}{|c|c|c|c|}
\hline Parameters & 1998-2006 & 2006-2009 & $p$ value \\
\hline survival & $24 / 33$ & $22 / 22$ & 0.01 \\
\hline enteral adaptation & $24 / 33$ & $18 / 22$ & NS \\
\hline $\begin{array}{l}\text { *mean time to adaptation } \\
\text { (months) }\end{array}$ & $4.5 \pm 3.1$ & $2.7 \pm 1.3$ & NS \\
\hline $\begin{array}{l}\text { time to death/follow up if } \\
\text { not adapted }\end{array}$ & $14.6 \pm 3.2$ & $16.6 \pm 12.8$ & NS \\
\hline $\begin{array}{l}\text { total time of follow up } \\
\text { (months) }\end{array}$ & $75 \pm 16$ & $15.4 \pm 8.0$ & 0.01 \\
\hline
\end{tabular}

33, O Fig. 1). Although the length of follow up was necessarily longer in the first cohort, most deaths occurred within the first 15 months of follow up. The cause of death in all cases was endstage liver failure and sepsis. In the only older child in the 19982006 cohort who died after 3 years of PN, liver failure was associated with severe macrophage activation syndrome, thought to be induced by the soybean-derived lipid preparation.

The development of liver failure also differed significantly between the two groups ( 0 Fig. 2 ). In the treatment era from 1998-2006, the average direct bilirubin after 3 months of PN was $112 \pm 34 \mu \mathrm{M} / \mathrm{L}$; in the recent era it was $8 \pm 2 \mu \mathrm{M} / \mathrm{L}$ ( $\mathrm{p}<0.01$ by Student's t-test). No patients in the recent cohort developed direct bilirubin which stayed above $100 \mathrm{uM} / \mathrm{L}$, while in the $1998-2006$ cohort roughly $25 \%$ of all patients on PN for longer than 25 weeks developed this biochemical marker of liver failure. This difference was clearly related to the change in nutritional support and PN treatment in the recent cohort, as the groups were similar in age and diagnosis. No other changes with regard to surgical or medical aspects of therapy were made dur- 

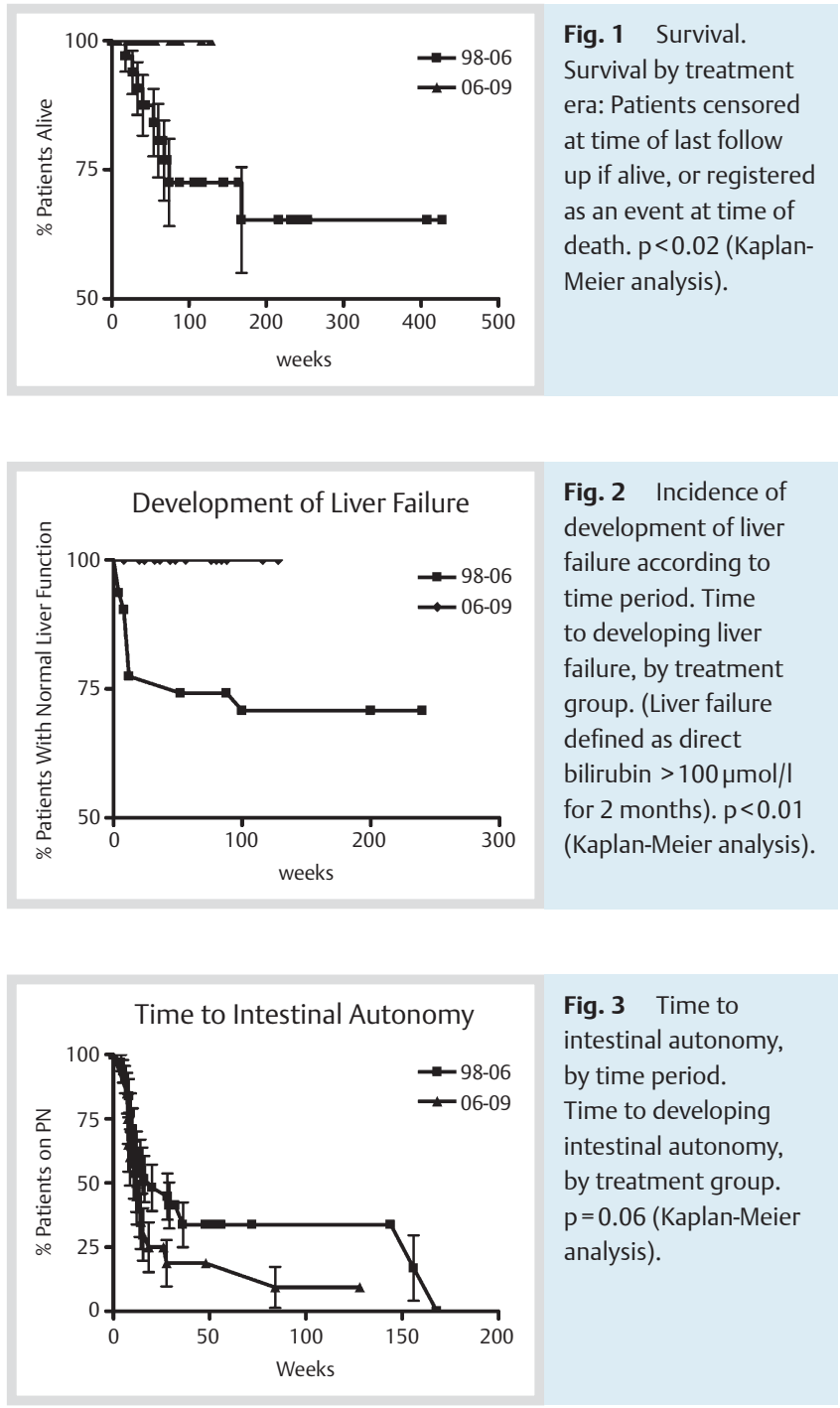

ing this time frame. The improvement in liver preservation does not appear to be due to an increased ability to induce intestinal adaptation and the corresponding ability to stop PN therapy. Although there was a reduction in the time required to achieving independence from PN in the recent cohort, the reduction was not statistically significantly different from the earlier time period ( $\bullet$ Fig. 3 ).

Finally, given the historical nature of the controls the average type of follow up is much longer in the 1998-2006 treatment group. However it is important to note that there was only one case of liver failure in this group which did not present in the first 6 months following the institution of PN therapy ( $\bullet$ Fig. 2). This was the unusual case of liver failure caused by macrophage activation described above. Thus, we do not anticipate that a significant number of the recent cohort is likely to develop liver failure after 6 months of successful therapy with PN; however, this will require ongoing follow up to confirm.

\section{Discussion}

$\nabla$

This study shows a distinct change in the incidence of liver failure and an improvement in survival in paediatric patients on long term parenteral nutrition from the initial cohort (19982006) and patients treated in the recent era, from 2006-2009.
These results support the utility of aggressive multidisciplinary treatment of intestinal failure with attention to bacterial overgrowth, and the modification of conventional parenteral nutrition using a lipid reduction/fish oil-based strategy if hyperbilirubinaemia occurs.

The observed improvement in long-term survival, as well as the improved preservation of liver function are the most important findings of this study ( $\bullet$ Figs. 1, 2; $\odot$ Table 3 ). The ability to preserve normal liver function over the long term has a number of critically important benefits for this patient population. Firstly, the cause of death in all the historical cohort patients was related to liver failure and sepsis. As noted, in one patient the development of liver failure was associated with macrophage activation syndrome, but the ultimate cause of death was liver failure and sepsis. All other cases had straightforward hepatic fibrosis and cirrhosis. This progressed to end-stage liver failure with ascites, poor hepatic synthetic function, and repeated cycles of bacterial and fungal sepsis. Several patients were listed for intestinal or combined intestinal-liver transplantation but did not receive grafts because of the lack of appropriate donors $[9,22,25]$.

The ability to maintain liver function in this patient population is critical to their survival, and quality of life. The time required for intestinal adaptation in these patients ranged from 4.5 months in the initial cohort to 2.7 months in the more recent cohort; these results are very similar to other recent reports in similar populations with mixed causes of post-surgical intestinal failure $[10,11,26]$. In the most severe cases of short bowel syndrome acquired in infancy, the time to successful adaptation averaged 2.6 years [8]. In our historical cohort, all patients in this category had succumbed to liver-related disease long before this adaptation would have been expected to occur ( $\bullet$ Table 3 ).

The improved outcome in the modern era was not related to the simple increase in the speed of adaptation; all of the 6 patients who were started on the lipid reduction/Omegevan therapy had rapid normalisation of liver function (within 10 weeks in all cases), despite the fact that only 2 of them came off PN in that time. Historically, resolution of PN-associated hyperbilirubinaemia typically takes 16 weeks, and only occurs after complete discontinuation of PN [13]. However, there may be some improvement in the rate of adaptation in the recent cohort of patients, as shown by the Kaplan-Meier curve of the time to reaching intestinal autonomy ( $\bullet$ Fig. $3 ; \mathrm{p}<0.06$ ).

In examining each of the elements of therapy in the modern era, the fundamental reasons for the improved outcome is not evident, and likely is to be due to a combination of factors. The use of continual drip feeds is likely important. Anecdotally we have observed that in situations where infants are managed with intermittent oral feeds or oral plus NG top up, as the volumes are slowly increased, the speed of adaptation seems to be somewhat slower than those managed with continuous NG feeds until full feeds are reached. The reasons for this are not clear, however it is interesting to note that observations in both the lab setting and in our human patients show that continuous feeds appear to result in a maximal stimulation of GLP-2 levels [27-29]. In turn, continuous elevation of GLP-2 levels have been shown to maximally induce adaptation $[28,29]$. To preserve oral feeding skills, we routinely start to encourage oral feeds when the infant is receiving more than $10 \mathrm{cc} / \mathrm{h}$ of EBM or formula, the feeds are held for $2 \mathrm{~h}$, and this volume is given by bottle with a gavage top up, twice daily. This maintains oral skills, and facilitates transitioning once the infant tolerates near full feeds Secondly, the use 
of rotating antibiotics has long been suggested to be useful in preventing PNALD [14]. The mechanism underlying this is not clear, however it has been postulated that ongoing bacterial overgrowth affects liver function via systemic, and especially portal, release of cytokines, this is turn may worsen the progress of liver disease $[13,18,29]$. This appears to have the greatest impact in infants younger than six months of age; older infants appear much less susceptible to this induced tendency to liver failure.

Overall, these results support the use of an aggressive pattern of rotating antibiotics in order to suppress small intestine bacterial overgrowth. This is particularly relevant because of the intentionally high enteral load of nutrients, in a condition of congenitally dilated, post-surgical alterations in motility, all of which will encourage the constitutive increase of small intestinal flora.

Fundamentally, the most likely cause of the improved outcomes was the use of lipid reduction/fish oil-based therapy. It is notable that while lipid reduction was used in infants whose direct bilirubin was increasing over $34 \mu \mathrm{mol} / \mathrm{L}$, this was not in itself adequate in the majority of cases [19]. Accordingly, if the bilirubin had not dropped significantly within 2-3 weeks, Omegevan therapy at $1 \mathrm{~g} / \mathrm{kg} /$ day was initiated [19]. The average time to resolution of hyperbilirubinaemia in these patients was 6.2 weeks; in 4 cases we were not able to advance enteral nutrition beyond $20 \%$ of total calories during that same time period. Thus we feel the benefit is primarily due to the use of the fish oilbased lipid preparation.

Importantly, we monitored the fatty acid profile using the serum triene:tetraene ratios. In 2 out of 3 long-term PN patients maintained exclusively with Omegevan for longer than 6 months, lipid profile abnormalities and clinical rashes suggestive of essential fatty acid deficiency, were detected. Once this was detected, the patients were supplemented with Intralipid as $50 \%$ of total IV fat and both the biochemical and clinical evidence of fatty acid deficiency improved; in the future we plan to switch to a combination of Intralipid/Omegevan after 6 months of PN. It is also important to note that while the cohort of patients treated with Omegevan had a dramatic normalisation of hepatic biochemistry and synthetic parameters, those patients who required long-term PN had persistent fibrosis on serial liver biopsies [30]. This has also been seen in other groups, and indicates the importance of continuing to aggressively increase enteral feeds, even in very short bowel syndrome patients; ultimately the only true "cure" for PNALD is discontinuation of PN $[9,17,18]$.

Finally, the STEP procedure was used in 4 patients in the recent era. This low incidence makes comments difficult; however, our experience is similar to that of other groups [20,21,31].

In these cases, each STEP procedure resulted in a significant improvement in the tolerance of enteral feeds; however, complete intestinal autonomy was only possible in 1 patient. It is important to note that in 2 of these patients a repeat STEP procedure is contemplated because of persistent recurrent dilation of the bowel. Thus we view the STEP procedure as a useful adjunct in the care of these patients. However, the fundamental drive to increase crypt cell proliferation may continue to cause intestinal dilation until the absorptive capacity is sufficient to maintain nutrition enterally [32].

\section{Limitations}

\section{$\nabla$}

This study suffers from the limitation of using historical cohorts. As shown in the demographic data, there has been a clear increase in patients presenting for intestinal failure as a result of gastroschisis ( Table 1). This reflects the worldwide increase in the number of babes born with gastroschisis [33,34]. In our population the prevalence of gastroschisis is 1 per 2400 live births. Importantly, the causes of intestinal failure, size, gestational age, and survival of this patient population are very similar to those in a number of recent reports $[1,8,10,11,17,35]$. Based on this, we suggest that the survival of the previous cohort of patients was similar to that seen in many other contemporary series; the finding of improved outcomes after the combination of a protocol-driven increase in enteral feeds, the use of rotating antibiotics, lipid reduction/fish oil-based lipid preparations and the STEP procedure may be a new standard for the care of this patient population. The results seen in this initial cohort are encouraging and justify continued evaluation.

\section{Conflict of interest: None}

\section{References}

1 Goulet $O$, Ruemmele $F$. Causes and management of intestinal failure in children. Gastroenterology 2006; 130: S16-S28

2 Wessel JJ, Kocoshis SA. Nutritional management of infants with short bowel syndrome. Semin Perinatol 2007; 31: 104-111

3 Hodes JE, Grosfeld JL, Weber TR et al. Hepatic failure in infants on total parenteral nutrition (TPN): Clinical and histopathologic observations. J Pediatr Surg 1982; 17: 463-468

4 Sigalet DL. Short bowel syndrome in infants and children: An overview. Semin Pediatr Surg 2001; 10: 49-55

5 Colomb V, Bbas-Tyan M, Taupin P et al. Long-term outcome of children receiving home parenteral nutrition: A 20-year single-center experience in 302 patients. J Pediatr Gastroenterol Nutr 2007; 44: 347-353

6 Koehler AN, Yaworski JA, Gardner M et al. Coordinated interdisciplinary management of pediatric intestinal failure: A 2-year review. J Pediatr Surg 2000; 35: 380-385

7 Schalamon J, Mayr JM, Hollwarth ME. Mortality and economics in short bowel syndrome. Best Pract Res Clin Gastroenterol 2003; 17: 931-942

8 Spencer $A U$, Kovacevich D, Kinney-Barnett $M$ et al. Pediatric shortbowel syndrome: The cost of comprehensive care. Am J Clin Nutr 2008; 88: 1552-1559

9 Kelly DA. Intestinal failure-associated liver disease: What do we know today? Gastroenterology 2006; 130: S70-S77

10 Wales PW, de Silva N, Kim J et al. Neonatal short bowel syndrome: Population-based estimates of incidence and mortality rates. J Pediatr Surg 2004; 39: 690-695

11 Wales PW, de Silva N, Kim JH et al. Neonatal short bowel syndrome: A cohort study. J Pediatr Surg 2005; 40: 755-762

12 DiBaise JK, Young RJ, Vanderhoof JA. Intestinal rehabilitation and the short bowel syndrome: Part 1. Am J Gastroenterol 2004; 99: 1386-1395

13 Javid PJ, Collier S, Richardson D et al. The role of enteral nutrition in the reversal of parenteral nutrition-associated liver dysfunction in infants. J Pediatr Surg 2005; 40: 1015-1018

14 Meehan JJ, Georgeson KE. Prevention of liver failure in parenteral nutrition-dependent children with short bowel syndrome. J Pediatr Surg 1997; 32: 473-475

15 Sigalet DL, Martin GR, Meddings JB. Methylglucose uptake as a marker of nutrient absorption and bowel length in pediatric patients. JPEN J Parenter Enteral Nutr 2004; 28: 158-162

16 Colomb V, Jobert-Giraud A, Lacaille F et al. Role of lipid emulsions in cholestasis associated with long-term parenteral nutrition in children. JPEN J Parenter Enteral Nutr 2000; 24: 345-350

17 Diamond IR, Sterescu A, Pencharz PB et al. Changing the paradigm: Omegevan for the treatment of liver failure in pediatric short bowel syndrome. J Pediatr Gastroenterol Nutr 2009; 48: 209-215 
18 Goulet $O$, Joly F, Corriol $O$ et al. Some new insights in intestinal failureassociated liver disease. Curr Opin Organ Transplant 2009; 14: 256-261

19 Gura KM, Lee S, Valim C et al. Safety and efficacy of a fish-oil-based fat emulsion in the treatment of parenteral nutrition-associated liver disease. Pediatrics 2008; 121: e678-e686

20 Javid PJ, Kim HB, Duggan CP et al. Serial transverse enteroplasty is associated with successful short-term outcomes in infants with short bowel syndrome. J Pediatr Surg 2005; 40: 1019-1023

21 Modi BP, Javid PJ, Jaksic T et al. First report of the international serial transverse enteroplasty data registry: Indications efficacy and complications. J Am Coll Surg 2007; 204: 365-371

22 Diamond IR, de SN, Pencharz PB et al. Neonatal short bowel syndrome outcomes after the establishment of the first Canadian multidisciplinary intestinal rehabilitation program: Preliminary experience. J Pediatr Surg 2007; 42: 806-811

23 Blau J, Sridhar S, Mathieson S et al. Effects of protein/nonprotein caloric intake on parenteral nutrition associated cholestasis in premature infants weighing 600-1000g. JPEN J Parenter Enteral Nutr 2007; 31 : 487-490

24 Struijs MC, Diamond IR, de SN et al. Establishing norms for intestinal length in children. J Pediatr Surg 2009; 44: 933-938

25 Fecteau A, Atkinson P, Grant D. Early referral is essential for successful pediatric small bowel transplantation: The Canadian experience. J Pediatr Surg 2001; 36: 681-684

26 Cole CR, Hansen NI, Higgins RD et al. Very low birth weight preterm infants with surgical short bowel syndrome: Incidence morbidity and mortality and growth outcomes at 18 and 22 months. Pediatrics 2008; 122: e573-e582

27 Amin H, Holst JJ, Hartmann B et al. Functional ontogeny of the proglucagon derived peptide axis in human neonates. Pediatrics 2008; 121 : e180-e186
28 Martin GR, Wallace LE, Sigalet DL. Glucagon-like peptide-2 induces intestinal adaptation in parenterally fed rats with short bowel syndrome. Am J Physiol Gastrointest Liver Physiol 2004; 286: G964-G972

29 Kaji T, Tanaka H, Holst JJ et al. The effects of variations in dose and method of administration on glucagon like peptide-2 activity in the rat. Eur J Pharmacol 2008; 596: 138-145

30 Fitzgibbons SC, Jones BA, Hull MA et al. The relationship between biopsy proven parenteral nutrition associated liver fibrosis and biochemical cholestasis in children with short bowel syndrome. J Pediatr Surg. In Press, 2009

31 Duggan C, Piper H, Javid PJ et al. Growth and nutritional status in infants with short-bowel syndrome after the serial transverse enteroplasty procedure. Clin Gastroenterol Hepatol 2006; 4: 1237-1241

32 Kaji T, Tanaka H, Wallace L et al. Nutritional effects of the serial transverse enteroplasty procedure in experimental short bowel syndrome. J Pediatr Surg 2009; 44: 1552-1559

33 Alvarez SM, Burd RS. Increasing prevalence of gastroschisis repairs in the United States: 1996-2003. J Pediatr Surg 2007; 42: 943-946

34 Skarsgard ED, Claydon J, Bouchard S et al. Canadian Pediatric Surgical Network: A population-based pediatric surgery network and database for analyzing surgical birth defects: The first 100 cases of gastroschisis. J Pediatr Surg 2008; 43: 30-34

35 Uauy RD, Fanaroff AA, Korones $S B$ et al. Necrotizing enterocolitis in very low birth weight infants: biodemographic and clinical correlates. National Institute of Child Health and Human Development Neonatal Research Network. J Pediatr 1991; 119: 630-638 